\title{
Detection of Low-Signal Pulvinar Areas Using Diffusion-Weighted Imaging in Patients with Dementia Experiencing Visual Hallucinations
}

\author{
Mayuko Sugiura $^{a}$ Masayuki Satoh $^{b}$ Ken-ichi Tabei $^{b, c}$ Tomoki Saito $^{a}$ \\ Mutsuki Moria Makiko Abe ${ }^{b}$ Hirotaka Kida $^{b}$ Masayuki Maedad \\ Hajime Sakuma ${ }^{e}$ Hidekazu Tomimotob, c \\ ${ }^{a}$ Faculty of Medicine, Mie University, and Departments of ${ }^{b}$ Dementia Prevention and \\ Therapeutics, ${ }^{c}$ Neurology, ${ }^{\mathrm{d}}$ Advanced Diagnostic Imaging and ${ }^{\mathrm{e}}$ Radiology, Graduate School \\ of Medicine, Mie University, Mie, Japan
}

\author{
Key Words \\ Dementia · Visual hallucinations · Pulvinar · 3T MRI · Diffusion-weighted image
}

\section{Abstract}

Background: Little research has been conducted regarding the role of pulvinar nuclei in the pathogenesis of visual hallucinations due to the difficulty of assessing abnormalities in this region using conventional magnetic resonance imaging (MRI). The present study aimed to retrospectively investigate the relative abilities of diffusion-weighted imaging (DWI), fluidattenuated inversion recovery (FLAIR), and susceptibility-weighted imaging (SWI) to visualize the pulvinar and to ascertain the relationship between pulvinar visualization and visual hallucinations. Methods: A retrospective analysis of 3T MRIs from 73 patients (31 males, 42 females; mean age $73.5 \pm 12.7$ years) of the Memory Clinic of Mie University Hospital was conducted. Correlations between pulvinar visualization and the following were analyzed: age, sex, education, hypertension, hyperlipidemia, diabetes mellitus, Mini-Mental State Examination score, Evans index, and visual hallucinations. Results: DWI detected low-signal pulvinar areas in approximately half of the patients (52.1\%). Participants with pulvinar visualization were significantly older, and the pulvinar was more frequently visualized in patients who had experienced visual hallucinations compared to those who had not. No significant association was observed between whole brain atrophy and pulvinar visualization. Conclusions: The results of the present study indicate that diffusion-weighted 3T MRI is the most suitable method for the detection of pulvinar nuclei in patients with dementia experiencing visual hallucinations. 
Sugiura et al.: Detection of Low-Signal Pulvinar Areas Using Diffusion-Weighted Imaging in Patients with Dementia Experiencing Visual Hallucinations

\section{Introduction}

Visual information transferred to the brain via the optic nerve mainly occurs through two distinct yet complementary pathways [1-3]. The majority of retinal axons terminate in the lateral geniculate nucleus, which relays information to the primary visual cortex (geniculate system); however, a second pathway allows some crude visual information to be transmitted to the cortex via the superior colliculus and pulvinar nucleus even when the optic radiation has been damaged (nongeniculate system). Damage to this latter pathway has been observed to result in both attentional/spatial deficits and neglect syndromes $[4,5]$.

Research involving both macaque monkeys and healthy humans has revealed that the pulvinar is involved in perceptual awareness and cognitive processing of optical illusions [6, 7]. Further, autopsy results have revealed that the nongeniculate system is more severely affected than the geniculate system in brains affected by dementia with Lewy bodies (DLB), indicating the potential involvement of the nongeniculate system in the production of visual hallucinations [8]. Additionally, there is a higher prevalence of pareidolia in DLB, wherein complex visual illusions involving ambiguous forms are perceived as meaningful objects [9]. Thus, researchers have suggested that the nongeniculate system is not only involved in the production of visual hallucinations but also in the processing of optical illusions. However, little research has focused on the role of the pulvinar in the pathogenesis of visual hallucinations, possibly due to the difficulties in visualizing pulvinar abnormalities using conventional MRI.

Moon et al. [10] reported that 3T MRIs of the fluid-attenuated inversion recovery (FLAIR) hypointensity of the pulvinar was more prominent in Alzheimer's disease (AD) subjects than in the age-matched control subjects. The pulvinar-to-corpus callosum signal intensity ratio was positively correlated with that of the Mini-Mental State Examination (MMSE) score. They concluded that the FLAIR hypointensity of the pulvinar nucleus represents an abnormal iron accumulation in $\mathrm{AD}$ and may be used as an adjunctive finding for evaluating AD.

We hypothesized that the hypointensity of the pulvinar nucleus using conventional MRI represents the pathogenesis of visual hallucinations in dementia-related diseases. As the pulvinar nucleus correlates visual processing, it may represent visual hallucinations rather than a decline in intellectual function. Visualization of the pulvinar using conventional MRI may help to elucidate the role of the pulvinar in the pathogenesis of visual hallucinations in neurodegenerative disorders.

In the present study, we retrospectively analyzed 3T MRIs from patients who presented to the Memory Clinic of Mie University Hospital in order to determine the relative capabilities of DWI, FLAIR, and susceptibility-weighted imaging (SWI) to visualize the pulvinar nuclei. SWI was chosen over T2-weighted imaging due to its greater sensitivity in the detection of iron depositions [11]. The present study additionally investigated the relationship between the presence of visual hallucinations and pulvinar visualization.

\section{Participants and Methods}

\section{Participants}

Seventy-three patients (31 males, 42 females; mean age $73.5 \pm 12.7$ years) who presented to the Memory Clinic of Mie University Hospital between January 2014 and June 2014 were recruited to participate in the present study. Forty-two patients had been diagnosed with AD, 13 with DLB, 7 with vascular dementia, and 9 with other forms of dementia. The remaining 2 patients had received no dementia diagnosis.

Diagnoses for AD were made according to the criteria established by the National Institute of Neurologic Disorders and Stroke/Alzheimer Disease and Related Disorders Association 


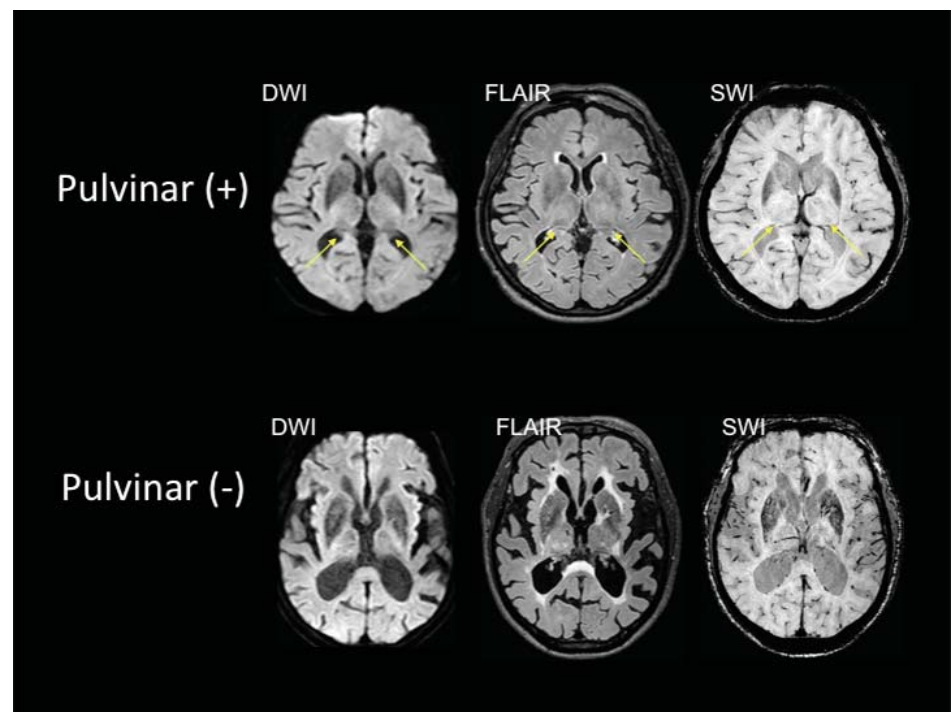

Fig. 1. Typical DWI, FLAIR, and SWI on which the pulvinar is visible (upper row; arrows) or invisible (lower row).

(NINCDS-ADRDA) [12]. Diagnoses of vascular dementia were made according to the criteria established by the National Institute of Neurologic Disorders and Stroke/Association Internationale pour la Recherche et l'Enseignement en Neurosciences (NINDS-AIREN) [13]. Diagnoses of DLB were made according to clinical criteria established by the consortium on DLB [14]. The present study was approved by the Ethical Review Board of Mie University Hospital, and all participants provided informed consent.

\section{MRI Protocol}

MRI was performed using an 8- or 32-channel phased-array head coil in a 3T MRI scanner (Achieva or Ingenia, Philips Medical System, The Netherlands). DWI, 3D-FLAIR, and SWI sequences were used to visualize the pulvinar nuclei.

DWIs were obtained using the following parameters: repetition time (TR) $=4,400 \mathrm{~ms}$; echo time $(\mathrm{TE})=87 \mathrm{~ms}$; slice thickness $=5 \mathrm{~mm}$; spacing $=0 \mathrm{~mm}$; b value $=1,000 \mathrm{~s} / \mathrm{mm}^{2} ;$ and field of view $(\mathrm{FOV})=230 \mathrm{~mm}$. FLAIR images were obtained using the following parameters: $\mathrm{TR}=6,000 \mathrm{~ms} ; \mathrm{TE}=400 \mathrm{~ms}$; section thickness $=1 \mathrm{~mm}$ with $0.5 \mathrm{~mm}$ overlap; and FOV $=60$ $\mathrm{mm}$; matrix $=288 \times 288$. SWIs were obtained using the following parameters: $\mathrm{TR}=22 \mathrm{~ms}$; $\mathrm{TE}=11.5 \mathrm{~ms}$; section thickness $=0.5 \mathrm{~mm}$ with overcontiguous slices; and FOV $=230 \mathrm{~mm}$; matrix $=320 \times 251$. All images were evaluated in an axial plane using 5-mm-slice thickness.

\section{Procedure}

Five reviewers chosen by the authors of the present study were asked to determine the presence or absence of pulvinar hypointensity in the MRIs of enrolled patients and to manually measure Evans indices [15] using hospital electronic medical records. The MMSE was also administered. Correlations were then analyzed between pulvinar visualization on MRIs and the following: age, sex, education, hypertension, hyperlipidemia, diabetes mellitus, MMSE score, Evans index, and visual hallucinations.

\section{Statistical Analyses}

Statistical analyses were conducted using independent $t$ tests for continuous variables, $\chi^{2}$ tests, and Fisher's exact test for dichotomous variables, as well as Mann-Whitney U tests for nonparametric data. Furthermore, we performed logistic regression analysis using a 
Fig. 2. Rates of pulvinar visualization. Pulvinar visualization by at least one modality occurred in 45 of 71 patients $(63.4 \%)$.

Table 1. Clinical characteristics of participants experiencing visual hallucinations

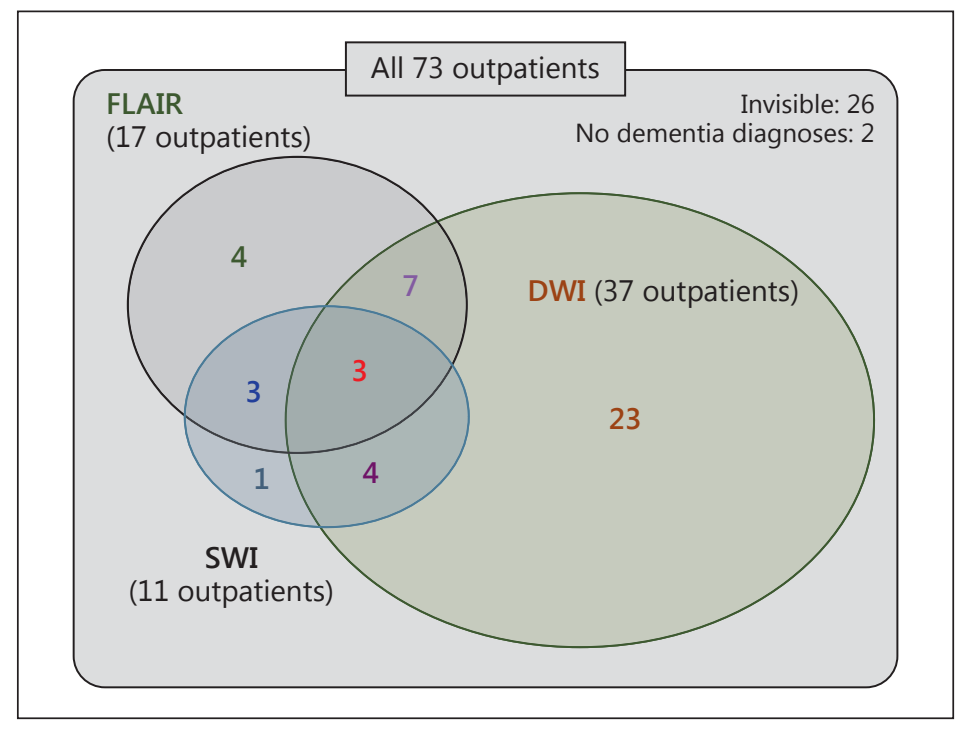

\begin{tabular}{lllll}
\hline $\begin{array}{l}\text { Patient } \\
\text { No. }\end{array}$ & $\begin{array}{l}\text { Clinical } \\
\text { diagnosis }\end{array}$ & $\begin{array}{l}\text { Age, years/ } \\
\text { Sex }\end{array}$ & $\begin{array}{l}\text { MMSE } \\
\text { score }\end{array}$ & $\begin{array}{l}\text { Pulvinar } \\
\text { visualization }\end{array}$ \\
\hline 1 & AD & $87 / \mathrm{F}$ & 11 & DWI, FLAIR \\
2 & AD & $91 / \mathrm{F}$ & 12 & DWI \\
3 & DLB & $87 / \mathrm{F}$ & 11 & FLAIR \\
4 & DLB & $78 / \mathrm{F}$ & n.s. & DWI, FLAIR, SWI \\
5 & DLB & $60 / \mathrm{M}$ & 24 & DWI, FLAIR \\
6 & DLB & $74 / \mathrm{F}$ & 21 & DWI, FLAIR, SWI \\
7 & DLB & $81 / \mathrm{F}$ & 21 & FLAIR, SWI \\
8 & PDD & $78 / \mathrm{F}$ & 23 & DWI \\
9 & DLB & $78 / \mathrm{M}$ & n.s. & DWI \\
10 & DLB & $82 / \mathrm{M}$ & n.s. & not visualized \\
\hline
\end{tabular}

PDD = Parkinson's disease with dementia; n.s. = not stated

generalized linear model. p values $<0.05$ were regarded as statistically significant. All analyses were performed using IBM SPSS Statistics version 20.0 (Chicago, Ill., USA).

\section{Results}

The 2 participants without dementia were excluded from analysis. Examples of DWIs, FLAIR, and SWIs are presented in figure 1 . The pulvinar was detected by at least one modality in 45 patients (63.4\%) (fig. 2). The pulvinar was more frequently visible on DWIs (37 patients) than with FLAIR (17 patients; $p=0.009$ ) and SWI (11 patients; $p<0.001$ ). The pulvinar regions of 3 patients were visualized in all modalities (fig. 1, upper row).

Clinical characteristics of participants who have experienced hallucinations are shown in table 1. We examined differences in age, MMSE score, and Evans index between individuals with or without pulvinar visualization. Although patients with visible pulvinar areas were significantly older than patients without, no significant difference was observed between the 
Table 2. Relationship between pulvinar visualization and patient characteristics

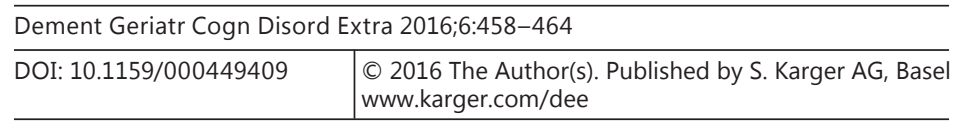

Sugiura et al.: Detection of Low-Signal Pulvinar Areas Using Diffusion-Weighted Imaging in Patients with Dementia Experiencing Visual Hallucinations

\begin{tabular}{llll}
\hline & $\begin{array}{l}\text { Pulvinar } \\
\text { visualization } \\
(\mathrm{n}=45)\end{array}$ & $\begin{array}{l}\text { No visualization } \\
(\mathrm{n}=26)\end{array}$ & $\mathrm{p}$ value \\
\hline Age, years & $75.2 \pm 12.2$ & $70.8 \pm 13.5$ & 0.017 \\
Sex, male/female & $19 / 26$ & $11 / 15$ & 0.99 \\
Education, years & $11.1 \pm 2.7$ & $11.8 \pm 2.6$ & 0.39 \\
Hypertension, \% & 47 & 27 & 0.10 \\
Hyperlipidemia, \% & 16 & 4 & 0.14 \\
Diabetes mellitus, \% & 20 & 12 & 0.36 \\
MMSE score & $22.0 \pm 5.6$ & $23.9 \pm 4.1$ & 0.19 \\
Evans index, \% & $25.3 \pm 4.3$ & $25.9 \pm 4.2$ & 0.61 \\
\hline
\end{tabular}

Table 3. Relationship between pulvinar visualization and presence of visual hallucinations

\begin{tabular}{lll}
\hline & \multicolumn{2}{l}{ Pulvinar } \\
\cline { 2 - 3 } & visible $(\mathrm{n}=45)$ & invisible $(\mathrm{n}=26)$ \\
\hline Visual hallucination (+) & 9 & 1 \\
Visual hallucination (-) & 36 & 25 \\
\hline
\end{tabular}


Sugiura et al.: Detection of Low-Signal Pulvinar Areas Using Diffusion-Weighted

Imaging in Patients with Dementia Experiencing Visual Hallucinations

hallucinations in patients with DLB. The results of the present and previous studies indicate that both the occipital lobe and pulvinar may be related to visual hallucinations.

The mechanism underlying the ability of DWI to more frequently detect the pulvinar than FLAIR or SWI remains uncertain. Moon et al. [10] reported that T2*-weighted images of the pulvinar nucleus might depict iron accumulation. An iron accumulation is visualized as areas of low-signal intensity on DWI, with even lower-signal intensities observed using SWI. However, the results of the present study indicate that SWI detected the pulvinar in less than one-third of patients when compared to DWI, suggesting that areas of low-signal intensity on DWI may not be due to iron accumulation. Though the mechanism of the pulvinar visualization in DWI remains to be clarified, DWI appears the most suitable method for visualizing intrinsic tissue factors and/or the local environment of the pulvinar region.

The present study has several limitations with regard to quantitative analysis. As this was a retrospective analysis, the amount of clinical data was quite limited, particularly with regard to the number of images obtained. Further investigation of the visual recognition capabilities of patients and interviews with caregivers regarding behavioral and psychological symptoms of dementia may elucidate the possible role of the pulvinar in cognitive and psychological processing. Further, while the present study utilized the Evans index to investigate the degree of brain atrophy, voxel-based morphometry may more clearly indicate anatomical differences between patients with or without pulvinar visualization. As the volume of the pulvinar could not be measured in the present study, the volume ratio between pulvinar and visual association areas may be associated with visual hallucinations. As there were no normal controls in the present study, we needed to investigate that the pulvinar nuclei are more likely to be visible or invisible. Future studies should involve prospective analysis using cognitive and anatomical procedures that are more elaborate to examine a larger number of participants both with and without dementia.

In conclusion, the results of the present study indicate that DWI is the most effective method for visualization of the pulvinar and that the rate of visualization is higher in patients experiencing visual hallucinations than in those with no history of hallucinations.

\section{References}

1 Underleider LG, Mishkin M: Two cortical visual systems; in Ingle DJ, Goodale MA, and Mansfield RJW (eds): Analysis of Visual Behavior. Cambridge, MIT Press, 1982, pp 549-586.

-2 Goodale MA, Milner AD: Separate visual pathways for perception and action. Trends Neurosci 1992;15:20-25.

-3 Lyon DC, Nassi JJ, Callaway EM: A disynaptic relay from superior colliculus to dorsal stream visual cortex in macaque monkey. Neuron 2010;65:270-279.

-4 Snow JC, Allen HA, Rafal RD, Humphreys GW: Impaired attentional selection following lesions to human pulvinar: evidence for homology between human and monkey. Proc Natl Acad Sci USA 2009;106:4054-4059.

-5 Arend I, Rafal R, Ward R: Spatial and temporal deficits are regionally dissociable in patients with pulvinar lesions. Brain 2008;131:2140-2152.

6 Wilke M, Mueller KM, Leopold DA: Neural activity in the visual thalamus reflects perceptual suppression. Proc Natl Acad Sci USA 2009;106:9465-9470.

7 Tabei K, Satoh M, Kida H, Kizaki M, Sakuma H, Sakuma H, Tomimoto H: Involvement of the extrageniculate system in the perception of optical illusions: a functional magnetic resonance imaging study. PLoS One 2015; 10:e0128750.

-8 Yamamoto R, Iseki E, Murayama N, Minegishi M, Marui W, Togo T, Katsuse O, Kato M, Iwatsubo T, Kosaka K, Arai H: Investigation of Lewy pathology in the visual pathway of brains of dementia with Lewy bodies. J Neurol Sci 2006;246:95-101.

-9 Uchiyama M, Nishio Y, Yokoi K, Hirayama K, Imamura T, Shimomura T, Mori E: Pareidolias: complex visual illusions in dementia with Lewy bodies. Brain 2012;135:2458-2469.

10 Moon WJ, Kim HJ, Roh HG, Choi JW, Han SH: Fluid-attenuated inversion recovery hypointensity of the pulvinar nucleus of patients with Alzheimer disease: its possible association with iron accumulation as evidenced by the $\mathrm{t} 2\left(^{*}\right)$ map. Korean J Radiol 2012;13:674-683. 
Sugiura et al.: Detection of Low-Signal Pulvinar Areas Using Diffusion-Weighted Imaging in Patients with Dementia Experiencing Visual Hallucinations

11 Mittal S, Wu Z, Neelavalli J, Haacke EM: Susceptibility-weighted imaging: technical aspects and clinical applications, part 2. AJNR Am J Neuroradiol 2009;30:232-252.

-12 McKhann G, Drachman D, Folstein M, Katzman R, Price D, Stadlan EM: Clinical diagnosis of Alzheimer's disease: report of the NINCDS-ADRDA Work Group under the auspices of Department of Health and Human Services Task Force on Alzheimer's Disease. Neurology 1984;34:939-944.

-13 Román GC, Tatemichi TK, Erkinjuntti T, Cummings JL, Masdeu JC, Garcia JH, Amaducci L, Orgogozo JM, Brun A, Hofman A, et al: Vascular dementia: diagnostic criteria for research studies. Report of the NINDS-AIREN International Workshop. Neurology 1993;43:250-260.

14 McKeith IG, Dickson DW, Lowe J, Emre M, O’Brien JT, Feldman H, et al: Diagnosis and management of dementia with Lewy bodies: third report of the DLB Consortium. Neurology 2005;65:1863-1872.

15 Evans WA: An encephalographic ratio for estimating ventricular enlargement and cerebral atrophy. Arch Neurol Psychiatr 1942;47:931-937.

16 Mori S: Responses to donepezil in Alzheimer's disease and Parkinson's disease. Ann NY Acad Sci 2002;977: 493-500.

-17 Satoh M, Ishikawa H, Meguro K, Kasuya M, Ishii H, Yamaguchi S: Improved visual hallucination by donepezil and occipital glucose metabolism in dementia with Lewy bodies: the Osaki-Tajiri project. Eur Neurol 2010;64: 337-344. 